\title{
Efficacy Intravenous Tranexamic Acid in Reducing Blood Loss after Elective Cesarean Sections
}

\author{
MOHAMED A. MOHAMED, M.D.; ADEL A. EL-BOGHDADY, M.D. and ESLAM G.F. HEGAZY, M.Sc. \\ The Department of Obstetrics and Gynecology, Faculty of Medicine Al-Azhar University
}

\begin{abstract}
Background: Delivery by CS can cause more complications than normal vaginal delivery and one of the most common complications is primary or secondary postpartum haemorrhage. The coagulation and fibrinolytic systems are believed to be in a state of dynamic balance that maintains an intact vascular system. Tranexamic acid (TXA) is a potent anti fibrinolytic agent that exerts its effects by blocking lysine binding sites on plasminogen molecules and has the potential to enhance the effectiveness of the patient's own haemostatic mechanisms.
\end{abstract}

Aim of Study: To study the efficacy and safety of tranexamic acid in reducing blood loss during and after the lower segment cesarean section.

Methods: This Prospective Comparative study was held on one hundred and fifty patients from Obstetrics and Gynaecology Department of El-Sayed Galal University Hospital (Al-Azhar University) and El-Sahel Teaching Hospital.

- Group A (TA group): 50 patients would had $1 \mathrm{~g} / 10 \mathrm{~mL}$ TA diluted with $20 \mathrm{~mL}$ of $5 \%$ glucose.

- Group B (Misoprostol group): 50 patients would had 5 rectal 200 micrograms Misoprostol pills (misotac) were used.

- Group C (Oxytocin group): 50 patients would had 20IU oxytocin in $500 \mathrm{~mL}$ lactated Ringer's solution will be infused at a rate of $125 \mathrm{~mL} / \mathrm{h}$

Results: No statically differences between groups as regarding demographic characteristics of the patients.No statistically significant difference between groups according to indication of CS. No statistically significant difference between groups according to vital signs before treatment. Statistically significant difference between groups according to vital signs immediately after placental delivery and $1 \mathrm{hr}$ after CS for oxytocin group. No statistically significant difference between groups according to vital signs ( $2 \mathrm{hr}$ after $\mathrm{CS}$ ). No statistically significant difference between groups according to laboratory data before delivery. No statistically significant difference between groups according to Total blood loss. No statistically significant difference between groups according to laboratory data on ( 2 nd day). No statistically significant difference between groups according to neonatal manifestations.

Correspondence to: Dr. Mohamed A. Mohamed, The Department of Obstetrics and Gynecology, Faculty of Medicine Al-Azhar University
Conclusion: The use of tranexamic acid prior to caesarean section may have the effectiveness to reduce and minimize blood loss with no major side effects recorded throughout the study either for the mother nor for the babyagainst postpartum hemorrhage as shown by the results of this study.

Key Words: Postpartum Hemorrhage (PPH) - Tranexamic Acid (TA) - Cesarean Section (CS).

\section{Introduction}

DELIVERY by CS can cause more complications than normal vaginal delivery and one of the most common complications is primary or secondary postpartum haemorrhage (20\%) [1] .

The incidence of cesarean delivery is increasing, and the average blood loss during cesarean delivery $(1000 \mathrm{~mL})$ is double the amount lost during vaginal delivery $(500 \mathrm{~mL})$ [2].

The hematocrit falls by $10 \%$ and blood transfusion is required in $6 \%$ of women undergoing cesarean delivery compared with $4 \%$ of women who have a vaginal birth. Numerous methods for performing cesarean section (CS) exist; the aim is a safe delivery for the infant with minimum maternal morbidity. Operative morbidity includes hemorrhage, anemia, blood transfusion, and the risks associated with receiving donor blood products. In severe cases, CS may result in major obstetric hemorrhage, hysterectomy, admission to an intensive care unit, or maternal death. Medications, such as oxytocin, misoprostol, prostaglandin F2a, and methylergonovine have been used to control bleeding after CS [3] .

In the haemostatic process, coagulation occurs rapidly at the site of a damaged vessel by the buildup of a tight net of fibrin; at the same time, the fibrinolytic system removes the fibrin deposits that might cause permanent vascular occlusion once vascular repair has taken place [4]

The coagulation and fibrinolytic systems are believed to be in a state of dynamic balance that 
maintains an intact vascular system [5]. Tranexamic acid (TXA) is a potent anti fibrinolytic agent that exerts its effects by blocking lysine binding sites on plasminogen molecules and has the potential to enhance the effectiveness of the patient's own haemostatic mechanisms. Consequently, clot breakdown (fibrinolysis) is inhibited and bleeding is reduced [6]. TA may enhance the effectiveness of the patient's own hemostatic mechanism [7].

TA has been used to reduce blood loss and the need for allogeneic blood transfusion in cardiac surgery, liver transplantation, and orthopedic surgical procedures, with variable results [7]. In gynecology and obstetrics, TA is most commonly used to treat idiopathic menorrhagia and is an effective and well-tolerated treatment when administered orally [9]. Bleeding associated with pregnancy (placental abruption, placenta previa) has also been treated with TA [7]

During delivery, when the placenta separates from the uterine wall, physiologic and haemostatic changes occur sequentially to reduce bleeding: Strong myometrial contractions, increased platelet activity, massive release of coagulation factors and consequently a parallel increase in fibrinolytic activity [9].

While oxytocin administration enhances the first mechanism, TXA administration might be able to counter the latter and thus facilitate the haemostatic process. Finally, the association between the extent of the initial decrease in plasma fibrinogen and the subsequent severity of blood loss reported in women with early PPH [10]. Suggests that both the coagulation and fibrinolysis processes are implicated in the control of postpartum blood loss and further supports the hypothesis that TXA might be effective in PPH prevention. Accordingly, there is a clear theoretical rationale for the use of antifibrinolytic agents to reduce postpartum blood loss [11].

\section{Patients and Methods}

This is a prospective comparative study, includes 150 patients collected from Obstetrics and Gynaecology Department of El-Sayed Galal University Hospital (Al-Azhar University) and ElSahel Teaching Hospital, in period between August 2017 and May 2018.

They were selected according to Inclusion and Exclusion criteria:

- Inclusion criteria:

- Age >_18 years.

- Term $>38$ wk delivered by C.S.
- Multiple pregnancies, macrosomia, poly hydrominos.

- Pregnancy complications such as Gestinal HTN.

\section{- Exclusion criteria:}

- Severe Medical and Surgical Complications including the heart, liver and kidney, brain Disease and blood disorders (Autoimmune disease, Sickle cell disease, Severe hemorrhagic disease).

- Allergy to tranexamic acid.

- History of thrombo embolic disorders.

- Abnormal placenta: Such as Placenta Previa, Placenta Abruption, Placental Adhesions caused by repeated artificial abortions.

- Pre eclampsia, Eclampsia, HELLP syndrome.

- Administration of low-molecular-weight heparin or antiplatelet agents during the week before delivery.

After a written informed consent obtained from every patients. The study approved by Ethical Committee of Al-Azhar University, Faculty of Medicine.

Patients were distributed in three groups:

- Group A (TA group): 50 patients with $1 \mathrm{~g} / 10 \mathrm{~m}$ TA diluted with $20 \mathrm{~m}$ of $5 \%$ glucose.

- Group B (Misoprostol group): 50 patients with 5 rectal 200 micrograms Misoprostol pills (misotac) were used.

- Group C (Oxytocin group): 50 patients with 20IU oxytocin in 500m lactated Ringer's solution infused at a rate of $125 \mathrm{~mL} / \mathrm{h}$.

\section{Intervention:}

TA was slowly administered intravenously over a 5-minute period at least 10 minute prior to skin incision.

After delivery, the 3 groups received a 5IU intravenous bolus of preprepared oxytocin and an antibiotic, $1 \mathrm{~g}$ cefazolin diluted in $20 \mathrm{~mL}$ normal saline, and administered over a 5-minute period.

\section{Clinical observations and laboratory examinations:} Clinical observations:

- Vital Signs: Heart Rate (HR), Respiratory Rate (RR), Blood Pressure (BP), Were Checked Immediately after placental delivery and 1 and 2 hour after birth respectively.

- Extent ofpost partum hemorrhaging: The blood was measured by weigh and volume during two periods following placental delivery to the end 
of surgery and from the end of the operation to 2 hours after birth.

- Uterine contractility and placental separation.

- Neonatal resuscitation.

- Side effects caused by tranexamic acid.

\section{Laboratory examinations:}

- Complete Blood Count (CBC) and urine analysis before delivery and on the 2 nd day after delivery.

- Liver and renal function test were performed. 1 day before delivery and the 2 nd day after birth.

- Prothrombin time and activity were tested in the two group before delivery and the 2 nd day after birth.

Estimated blood loss was calculated using the difference in hematocrit values taken prior to and 48 hour after cesarean delivery, according to the following formula:

Estimated blood loss $=$

EBV x (Preop hematocrit Postop hematocrit)

Preop hematocrit

Where EBV (estimated blood volume) in $\mathrm{mL}$ $=$ The woman's weight in $\operatorname{kgx} 85$.

\section{Statistical analysis:}

Recorded data were analyzed using the statistical package for social sciences, version 20.0 (SPSS Inc., Chicago, Illinois, USA). Quantitative data were expressed as mean \pm standard deviation (SD). Qualitative data were expressed as frequency and percentage.

The following tests were done:

-A one-way analysis of variance (ANOVA) when comparing between more than two means.

- Post Hoc test: Least Significant Difference (LSD) was used for multiple comparisons between different variables.

- The confidence interval was set to $95 \%$ and the margin of error accepted was set to $5 \%$. So, the $p$-value was considered significant as the following:

- Probability ( $p$-value).

- $p$-value $<0.05$ was considered significant.

- $p$-value $<0.001$ was considered as highly significant.

- $p$-value $>0.05$ was considered insignificant.

\section{Results}

Table (1) shows no statistically significant difference between groups according to demographic data.
Table (1): Comparison between groups according to demographic data.

\begin{tabular}{|c|c|c|c|c|c|}
\hline $\begin{array}{l}\text { Demographic } \\
\text { data }\end{array}$ & $\begin{array}{c}\text { Group I: } \\
\text { TA } \\
(\mathrm{N}=50)\end{array}$ & $\begin{array}{l}\text { Group II: } \\
\text { Misoprostol } \\
(\mathrm{N}=50)\end{array}$ & $\begin{array}{c}\text { Group III: } \\
\text { Oxytocin } \\
(\mathrm{N}=50)\end{array}$ & $\mathrm{F}$ & $\begin{array}{c}p- \\
\text { value }\end{array}$ \\
\hline \multicolumn{6}{|l|}{ Age (years): } \\
\hline Range & $19-34$ & $20-34$ & $19-33$ & 1.074 & 0.182 \\
\hline \multirow[t]{2}{*}{ Mean \pm SD } & $26.50 \pm$ & $27.01 \pm$ & $26.11 \pm$ & & \\
\hline & 5.57 & 5.67 & 5.46 & & \\
\hline \multicolumn{6}{|l|}{ Weight $(\mathrm{kg})$ : } \\
\hline Range & $60-130$ & $65-123$ & $62-129$ & 0.482 & 0.874 \\
\hline \multirow[t]{2}{*}{ Mean \pm SD } & $95.91 \pm$ & $94.83 \pm$ & $96.46 \pm$ & & \\
\hline & 18.68 & 19.84 & 17.63 & & \\
\hline \multirow{2}{*}{\multicolumn{6}{|c|}{$\begin{array}{l}\text { Gestational age } \\
(w k s):\end{array}$}} \\
\hline & & & & & \\
\hline Range & $38-40$ & $38-40$ & $38-40$ & 0.117 & 0.214 \\
\hline \multirow[t]{2}{*}{ Mean \pm SD } & $39.15 \pm$ & $39.50 \pm$ & $39.25 \pm$ & & \\
\hline & 0.82 & 0.83 & 0.82 & & \\
\hline \multicolumn{6}{|l|}{ Parity: } \\
\hline Range & $0-5$ & $0-4$ & $0-5$ & $\mathrm{z}=1.115$ & 0.124 \\
\hline Median (IQR) & $2(2)$ & $2(1)$ & $2(1)$ & & \\
\hline
\end{tabular}

F: ANOVA test. $\quad$ z: Mann-Whitney test. $\quad p$-value $>0.05$ NS.

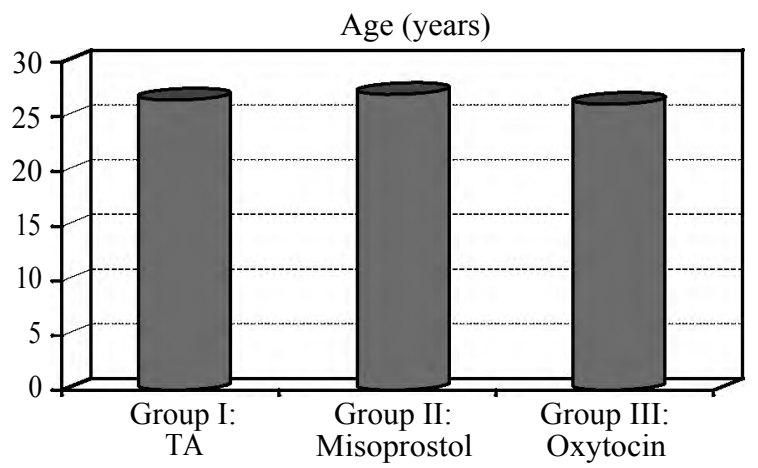

Fig. (1): Bar chart between groups according to age (years).

Table (2): Comparison between groups according to indication of CS.

\begin{tabular}{|c|c|c|c|c|c|}
\hline $\begin{array}{l}\text { Indication of } \\
\text { CS }\end{array}$ & $\begin{array}{l}\text { Group I: } \\
\text { TA } \\
(\mathrm{N}=50)\end{array}$ & $\begin{array}{l}\text { Group II: } \\
\text { Misoprostol } \\
(\mathrm{N}=50)\end{array}$ & $\begin{array}{l}\text { Group III: } \\
\text { Oxytocin } \\
(\mathrm{N}=50)\end{array}$ & $\begin{array}{l}\text { Chi- } \\
\text { square } \\
\text { test }\end{array}$ & $\begin{array}{c}p- \\
\text { value }\end{array}$ \\
\hline Elective CS & $27(54.0 \%)$ & $21(42.0 \%)$ & $22(44.0 \%)$ & \multirow{10}{*}{1.216} & \multirow{10}{*}{0.128} \\
\hline $\begin{array}{l}\text { Non reactive } \\
\text { CTG }\end{array}$ & $3(6.0 \%)$ & $4(8.0 \%)$ & $7(14.0 \%)$ & & \\
\hline $\begin{array}{l}\text { PG with breech } \\
\text { presentation }\end{array}$ & $2(4.0 \%)$ & $5(10.0 \%)$ & $4(8.0 \%)$ & & \\
\hline $\begin{array}{l}\text { Failure to } \\
\text { progression } \\
\text { in labour }\end{array}$ & $4(8.0 \%)$ & $4(8.0 \%)$ & $3(6.0 \%)$ & & \\
\hline $\begin{array}{l}\text { ROM with } \\
\text { meconium } \\
\text { stained liquor }\end{array}$ & $3(6.0 \%)$ & $6(12.0 \%)$ & $3(6.0 \%)$ & & \\
\hline Cord prolapse & $1(2.0 \%)$ & $0(0.0 \%)$ & $0(0.0 \%)$ & & \\
\hline $\begin{array}{l}\text { Multiple } \\
\text { pregnancy }\end{array}$ & $2(4.0 \%)$ & $1(2.0 \%)$ & $2(4.0 \%)$ & & \\
\hline $\begin{array}{l}\text { Gestational } \\
\text { hypertension }\end{array}$ & $3(6.0 \%)$ & $5(10.0 \%)$ & $5(10.0 \%)$ & & \\
\hline $\begin{array}{l}\text { Poly } \\
\text { hydrominos }\end{array}$ & $3(6.0 \%)$ & $1(2.0 \%)$ & $2(4.0 \%)$ & & \\
\hline Macrosomia & $2(4.0 \%)$ & $3(6.0 \%)$ & $2(4.0 \%)$ & & \\
\hline
\end{tabular}

$\mathrm{x}^{2}$ : Chi-square test.

Table (2) shows no statistically significant difference between groups according to indication of CS. 


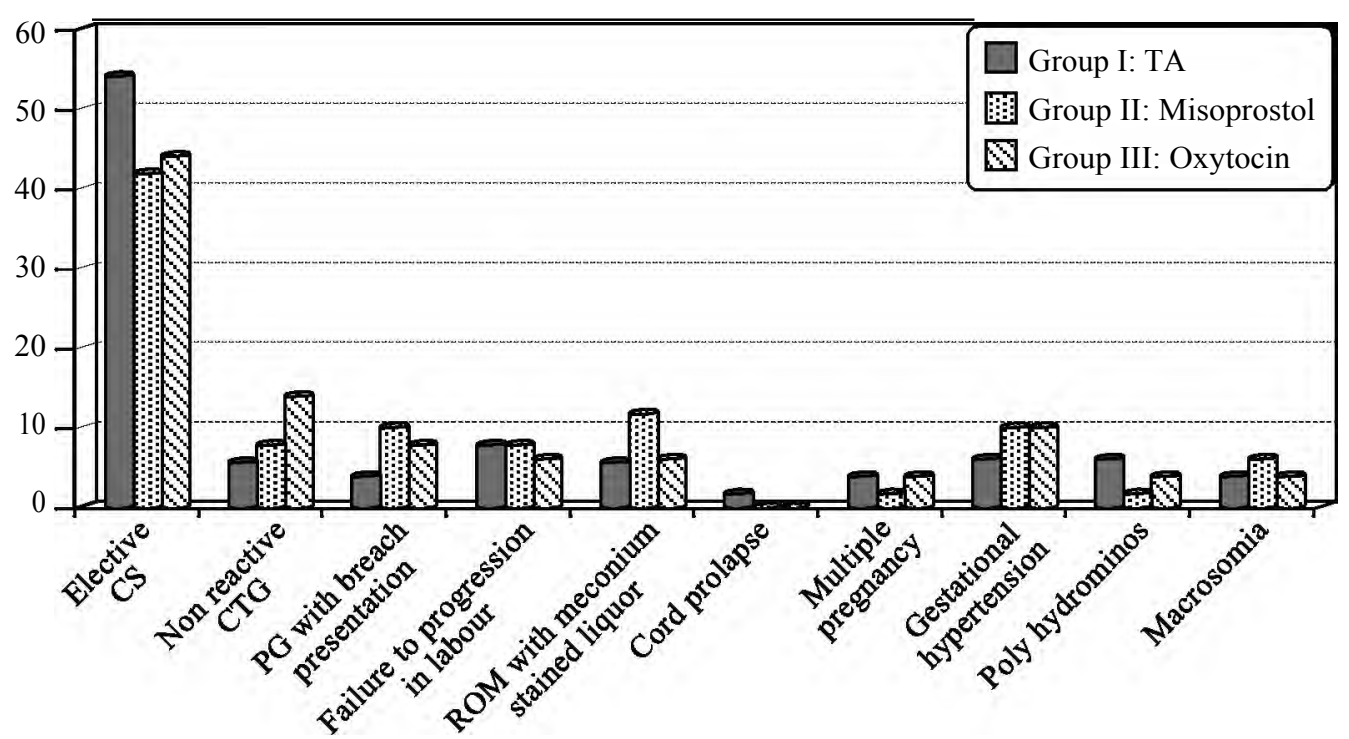

Fig. (2): Bar chart between groups according to indications of CS.

Table (3): Comparison between groups according to vital signs before treatment.

\begin{tabular}{|c|c|c|c|c|c|}
\hline $\begin{array}{l}\text { Vital signs } \\
\text { before treatment }\end{array}$ & $\begin{array}{c}\text { Group I: } \\
\text { TA } \\
(\mathrm{N}=50)\end{array}$ & $\begin{array}{c}\text { Group II: } \\
\text { Misoprostol } \\
(\mathrm{N}=50)\end{array}$ & $\begin{array}{c}\text { Group III: } \\
\text { Oxytocin } \\
(\mathrm{N}=50)\end{array}$ & $\mathrm{F}$ & $\begin{array}{c}p- \\
\text { value }\end{array}$ \\
\hline Systolic BP & $\begin{array}{l}121.20 \pm \\
10.91\end{array}$ & $\begin{array}{l}119.18 \pm \\
10.73\end{array}$ & $\begin{array}{l}123.22 \pm \\
11.09\end{array}$ & 0.827 & 0.290 \\
\hline Diastolic BP & $\begin{array}{l}75.75 \pm \\
6.82\end{array}$ & $\begin{array}{l}76.76 \pm \\
6.91\end{array}$ & $\begin{array}{l}74.74 \pm \\
6.73\end{array}$ & 0.306 & 0.445 \\
\hline Respiratory Rate & $\begin{array}{l}14.14 \pm \\
1.27\end{array}$ & $\begin{array}{l}13.13 \pm \\
1.18\end{array}$ & $\begin{array}{l}15.15 \pm \\
1.36\end{array}$ & 0.135 & 0.544 \\
\hline Heart Rate & $\begin{array}{l}85.85 \pm \\
7.73\end{array}$ & $\begin{array}{l}88.88 \pm \\
8.00\end{array}$ & $\begin{array}{l}87.87 \pm \\
7.91\end{array}$ & 0.337 & 0.490 \\
\hline
\end{tabular}

F: ANOVA test. $\quad p$-value $>0.05$ NS.

Table (3) shows no statistically significant difference between groups according to vital signs before treatment.

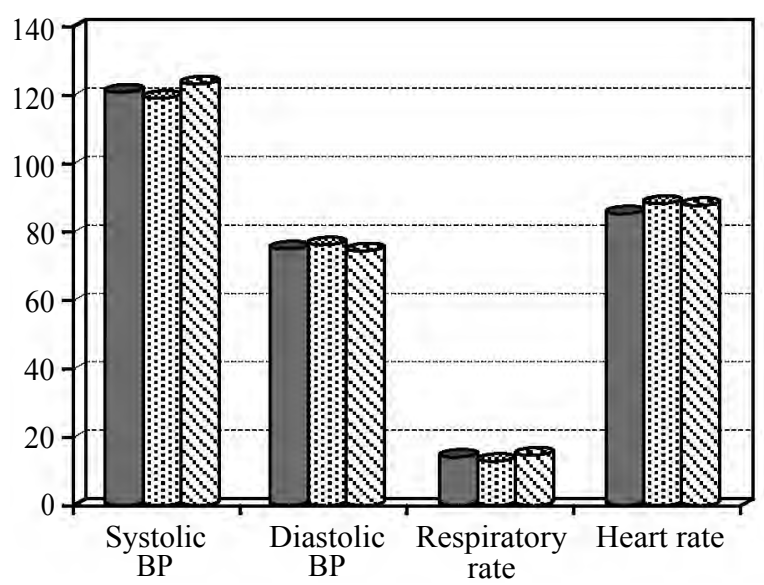

$$
\begin{aligned}
& \text { Group I: TA } \\
& \text { STroup II: Misoprostol } \\
& \text { S Group III: Oxytocin }
\end{aligned}
$$

Fig. (3): Bar chart between groups according to vital signs before treatment.
Table (4): Comparison between groups according to vital signs immediately after placental delivery.

\begin{tabular}{llllll}
\hline $\begin{array}{l}\text { Vital signs } \\
\text { immediately after } \\
\text { placental delivery }\end{array}$ & $\begin{array}{c}\text { Group I: } \\
\text { TA }\end{array}$ & $\begin{array}{c}\text { Group II: } \\
\text { Misoprostol Oxytocin } \\
(\mathrm{N}=50)\end{array}$ & $\begin{array}{c}\text { Group III: } \\
(\mathrm{N}=50)\end{array}$ & Test & $\begin{array}{c}p \text { - } \\
\text { value }\end{array}$ \\
\hline Systolic BP & $100 \pm$ & $107 \pm$ & $111 \pm$ & $3.4600 .021 *$ \\
& 9.30 & $9.95^{\mathbf{a}}$ & $10.32^{\mathbf{a}}$ & \\
Diastolic BP & $70.50 \pm$ & $71.44 \pm$ & $69.56 \pm$ & 0.3370 .490 \\
& 6.35 & 6.43 & 6.26 & \\
Respiratory Rate & $14.84 \pm$ & $13.78 \pm$ & $15.90 \pm$ & 0.1490 .598 \\
& 1.34 & 1.24 & 1.43 & & \\
Heart Rate & $95.20 \pm$ & $89.76 \pm$ & $88.74 \pm$ & 8.890 & $0.020^{*}$ \\
& 8.57 & $8.08^{\mathbf{a}}$ & $7.99 \mathbf{a}$ & & \\
\hline
\end{tabular}

a: Significant group I. $\quad$ b: Significant group II

F: ANOVA test. $\quad p$-value $>0.05 \mathrm{NS} . * p$-value $<0.05 \mathrm{~S}$.

Table (4) shows statistically significant difference between groups according to systolic and heart rate.

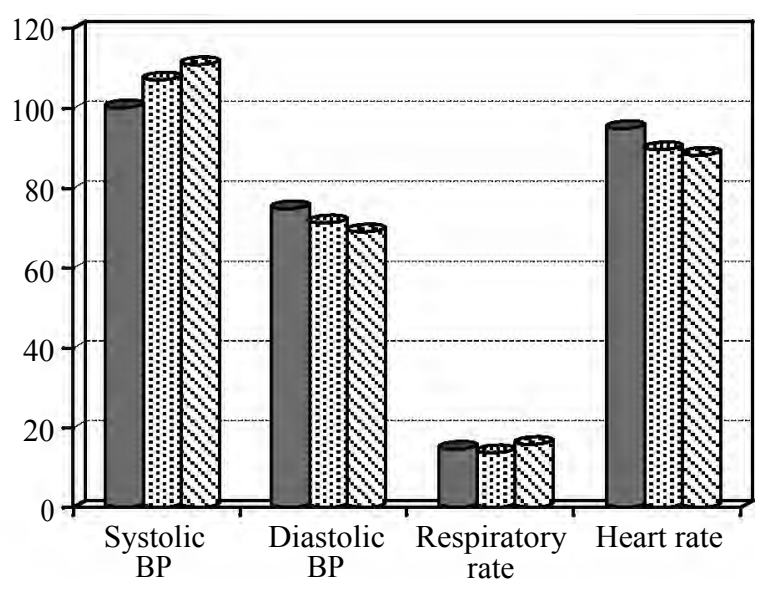

$$
\begin{aligned}
& \text { Group I: TA } \\
& \text { Troup II: Misoprostol } \\
& \text { Troup III: Oxytocin }
\end{aligned}
$$

Fig. (4): Bar chart between groups according to vital signs immediately after placental delivery. 
Table (5): Comparison between groups according to vital signs ( $1 \mathrm{hr}$ after CS).

\begin{tabular}{llllll}
\hline $\begin{array}{l}\text { Vital signs } \\
(1 \mathrm{hr} \text { after CS })\end{array}$ & $\begin{array}{c}\text { Group I: } \\
\text { TA } \\
(\mathrm{N}=50)\end{array}$ & $\begin{array}{c}\text { Group II: } \\
\text { Misoprostol Oxytocin } \\
(\mathrm{N}=50)\end{array}$ & $\begin{array}{c}\text { Group III: } \\
(\mathrm{N}=50)\end{array}$ & & $\begin{array}{c}p \text { - } \\
\text { value }\end{array}$ \\
\hline Systolic BP & $105 \pm$ & $116 \pm$ & $118 \pm$ & $3.8060 .023 *$ \\
& 9.45 & $10.44 \mathbf{a}$ & $10.62 \mathbf{a}$ & \\
Diastolic BP & $72.62 \pm$ & $73.58 \pm$ & $71.65 \pm$ & 0.3700 .538 \\
& 6.54 & 6.62 & 6.45 & \\
Respiratory Rate & $14.39 \pm$ & $13.37 \pm$ & $15.42 \pm$ & 0.1630 .658 \\
& 1.30 & 1.20 & 1.39 & & \\
Heart Rate & $93.30 \pm$ & $87.96 \pm$ & $86.97 \pm$ & 9.779 & $0.022^{*}$ \\
& 8.40 & $7.92 \mathbf{a}$ & $7.83^{\mathbf{a}}$ & & \\
\hline
\end{tabular}

a: Significant group I. $\quad$ b: Significant group II.

F: ANOVA test. $\quad p$-value $>0.05 \mathrm{NS} . \quad * p$-value $<0.05 \mathrm{~S}$.

Table (5) shows statistically significant difference between groups according to vital signs $(1 \mathrm{hr}$ after CS).
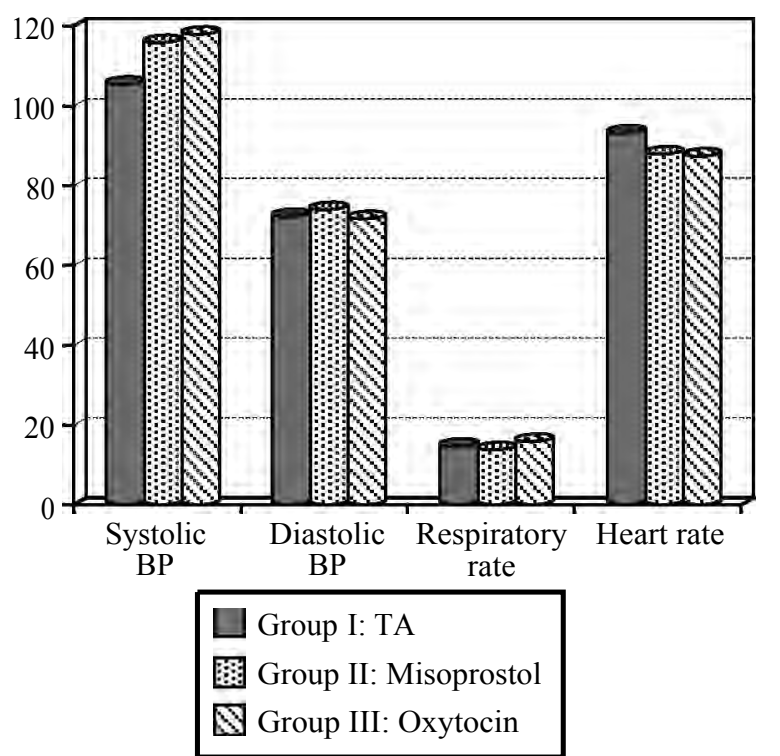

Fig. (5): Bar chart between groups according to vital signs (1hr after CS).

Table (6): Comparison between groups according to vital signs ( $2 \mathrm{~h}$ after $\mathrm{CS})$.

\begin{tabular}{llllll}
\hline $\begin{array}{l}\text { Vital signs } \\
(2 \mathrm{hr} \text { after CS })\end{array}$ & $\begin{array}{c}\text { Group I: } \\
\text { TA } \\
(\mathrm{N}=50)\end{array}$ & $\begin{array}{c}\text { Group II: } \\
\text { Misoprostol } \\
(\mathrm{N}=50)\end{array}$ & $\begin{array}{c}\text { Group III: } \\
\text { Oxytocin } \\
(\mathrm{N}=50)\end{array}$ & $\mathrm{F}$ & $\begin{array}{c}p \text { - } \\
\text { value }\end{array}$ \\
\hline Systolic BP & $117 \pm$ & $119 \pm$ & $123 \pm$ & 0.306 & 0.449 \\
& 10.49 & 10.75 & 11.04 & & \\
Diastolic BP & $74.79 \pm$ & $75.79 \pm$ & $73.80 \pm$ & 0.026 & 0.657 \\
& 6.73 & 6.82 & 6.64 & & \\
Respiratory Rate & $13.96 \pm$ & $12.97 \pm$ & $14.96 \pm$ & 0.186 & 0.581 \\
& 1.26 & 1.17 & 1.35 & & \\
Heart Rate & $86.77 \pm$ & $85.33 \pm$ & $84.36 \pm$ & 0.055 & 0.440 \\
& 7.81 & 7.68 & 7.59 & & \\
\hline
\end{tabular}

F: ANOVA test. $\quad p$-value $>0.05$ NS.

Table (6) shows no statistically significant difference between groups according to vital signs (2hr after CS).

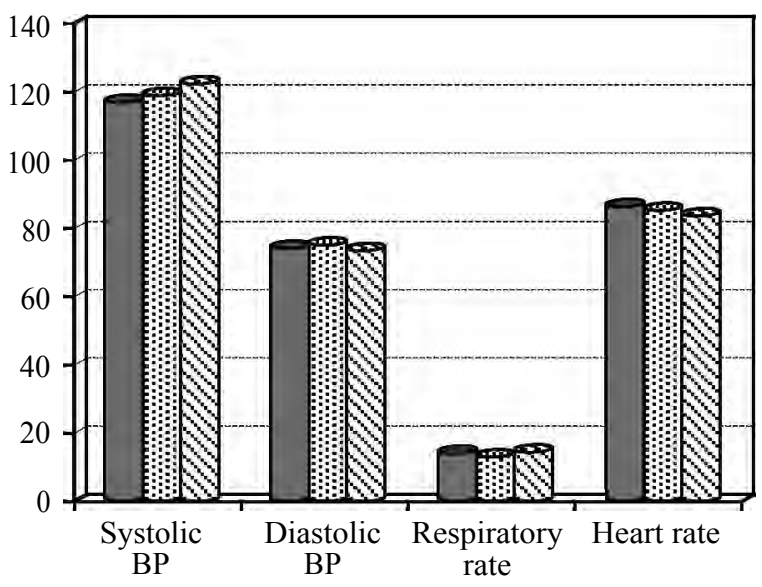

Group I: TA

鹳 Group II: Misoprostol

§ Group III: Oxytocin

Fig. (6): Bar chart between groups according to vital signs (2hr after CS).

Table (7): Comparison between groups according to \% of change in vital signs.

\begin{tabular}{|c|c|c|c|c|c|}
\hline $\begin{array}{l}\% \text { of change } \\
\text { in vital signs }\end{array}$ & $\begin{array}{c}\text { Group I: } \\
\text { TA } \\
(\mathrm{N}=50)\end{array}$ & $\begin{array}{l}\text { Group II: } \\
\text { Misoprostol } \\
(\mathrm{N}=50)\end{array}$ & $\begin{array}{c}\text { Group III: } \\
\text { Oxytocin } \\
(\mathrm{N}=50)\end{array}$ & $\mathrm{F}$ & $\begin{array}{c}p- \\
\text { value }\end{array}$ \\
\hline \multicolumn{6}{|l|}{$\begin{array}{l}\% \text { of change } \\
\text { before and after } \\
\text { placental: }\end{array}$} \\
\hline Systolic BP & $\begin{array}{l}20.00 \pm \\
6.20\end{array}$ & $\begin{array}{l}11.00 \pm \\
3.41 \mathbf{a}^{-}\end{array}$ & $\begin{array}{l}11.00 \pm \\
3.41 \mathrm{a}\end{array}$ & 7.774 & $0.013 *$ \\
\hline Diastolic BP & $\begin{array}{l}4.50 \pm \\
1.40\end{array}$ & $\begin{array}{l}4.56 \pm \\
1.41^{\mathrm{a}}\end{array}$ & $\begin{array}{l}4.44 \pm \\
1.38 \mathbf{a}\end{array}$ & 3.182 & $0.020 *$ \\
\hline Respiratory Rate & $\begin{array}{l}-0.84 \pm \\
0.26\end{array}$ & $\begin{array}{l}-0.78 \pm \\
0.24^{\mathbf{a}}\end{array}$ & $\begin{array}{l}-0.90 \pm \\
0.28 \mathbf{a}\end{array}$ & 4.239 & $0.017 *$ \\
\hline Heart Rate & $\begin{array}{l}-10.20 \pm \\
3.16\end{array}$ & $\begin{array}{l}-1.76 \pm \\
0.55 \mathbf{a}\end{array}$ & $\begin{array}{l}-1.74 \pm \\
0.54 \mathbf{a}\end{array}$ & 3.501 & $0.013 *$ \\
\hline \multicolumn{6}{|l|}{$\begin{array}{l}\text { \% of change } \\
\text { before and after } \\
\text { lhr: }\end{array}$} \\
\hline Systolic BP & $\begin{array}{l}15.00 \pm \\
4.65\end{array}$ & $\begin{array}{l}2.00 \pm \\
0.62 \mathbf{a}\end{array}$ & $\begin{array}{l}4.00 \pm \\
1.24 \mathbf{a}\end{array}$ & 7.307 & $0.015 *$ \\
\hline Diastolic BP & $\begin{array}{l}2.39 \pm \\
0.74\end{array}$ & $\begin{array}{l}2.42 \pm \\
0.75 \mathbf{a}\end{array}$ & $\begin{array}{l}2.35 \pm \\
0.73^{\mathbf{a}}\end{array}$ & 3.819 & $0.022 *$ \\
\hline Respiratory Rate & $\begin{array}{l}-0.39 \pm \\
0.12\end{array}$ & $\begin{array}{l}-0.37 \pm \\
0.11 \mathbf{a}^{ \pm}\end{array}$ & $\begin{array}{l}-0.42 \pm \\
0.13^{\mathbf{a}}\end{array}$ & 3.985 & $0.019 *$ \\
\hline Heart Rate & $\begin{array}{l}-8.30 \pm \\
2.74\end{array}$ & $\begin{array}{l}0.04 \pm \\
0.01\end{array}$ & $\begin{array}{l}0.03 \pm \\
0.01^{\mathbf{a}}\end{array}$ & 3.291 & $0.015^{*}$ \\
\hline \multicolumn{6}{|l|}{$\begin{array}{l}\% \text { of change } \\
\text { before and after } \\
2 \mathrm{hr} \text { : }\end{array}$} \\
\hline Systolic BP & $\begin{array}{l}3.45 \pm \\
1.14\end{array}$ & $\begin{array}{l}-1.48 \pm \\
0.49 \mathbf{a}\end{array}$ & $\begin{array}{l}-0.72 \pm \\
0.24 \mathbf{a}\end{array}$ & 6.869 & $0.014^{*}$ \\
\hline Diastolic BP & $\begin{array}{l}0.21 \pm \\
0.09\end{array}$ & $\begin{array}{l}0.21 \pm \\
0.09^{\mathbf{a}}\end{array}$ & $\begin{array}{l}0.20 \pm \\
0.09^{\mathbf{a}}\end{array}$ & 4.583 & $0.021 *$ \\
\hline Respiratory Rate & $\begin{array}{l}0.04 \pm \\
0.02\end{array}$ & $\begin{array}{l}0.03 \pm \\
0.01\end{array}$ & $\begin{array}{l}0.04 \pm \\
0.02^{\mathbf{a}}\end{array}$ & 3.746 & $0.018 *$ \\
\hline Heart Rate & $\begin{array}{l}-1.77 \pm \\
0.58\end{array}$ & $\begin{array}{l}2.67 \pm \pm \\
0.88\end{array}$ & $\begin{array}{l}2.64 \pm \\
0.87^{\mathbf{a}}\end{array}$ & 3.093 & $0.014 *$ \\
\hline
\end{tabular}

a: Significant group I. $\quad$ b: Significant group II.

F: ANOVA test. $\quad * p$-value $<0.05 \mathrm{~S}$.

Table (7) shows statistically significant difference between groups according to \% of change in vital signs. 


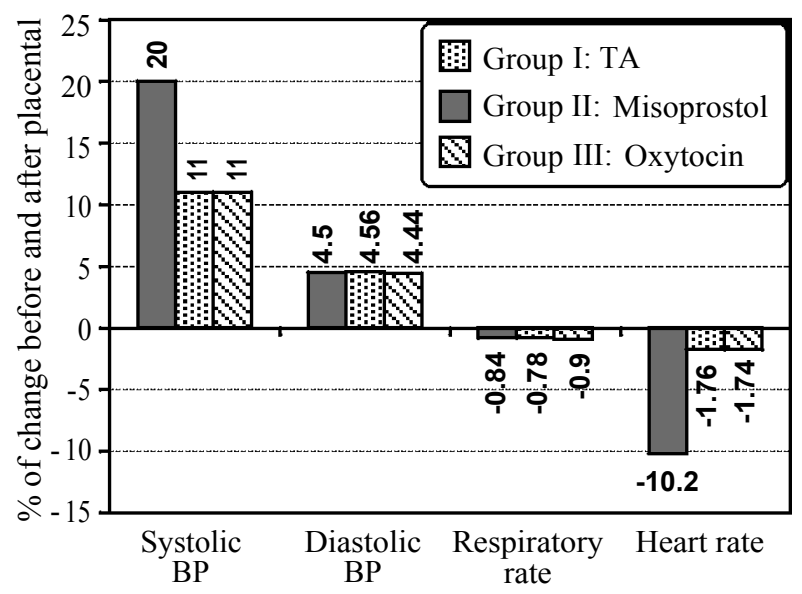

Fig. (7): Bar chart between groups according to \% of change before and after placental.

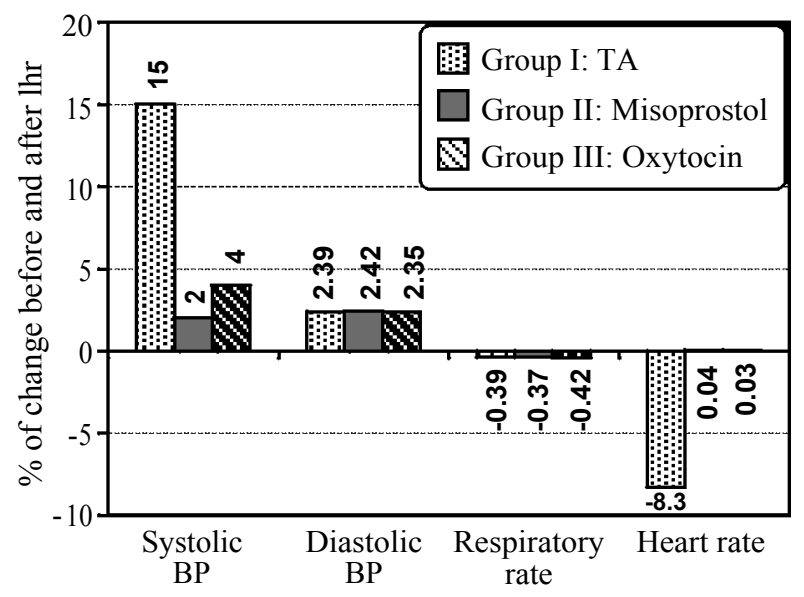

Fig. (8): Bar chart between groups according to $\%$ of change before and after $1 \mathrm{hr}$.

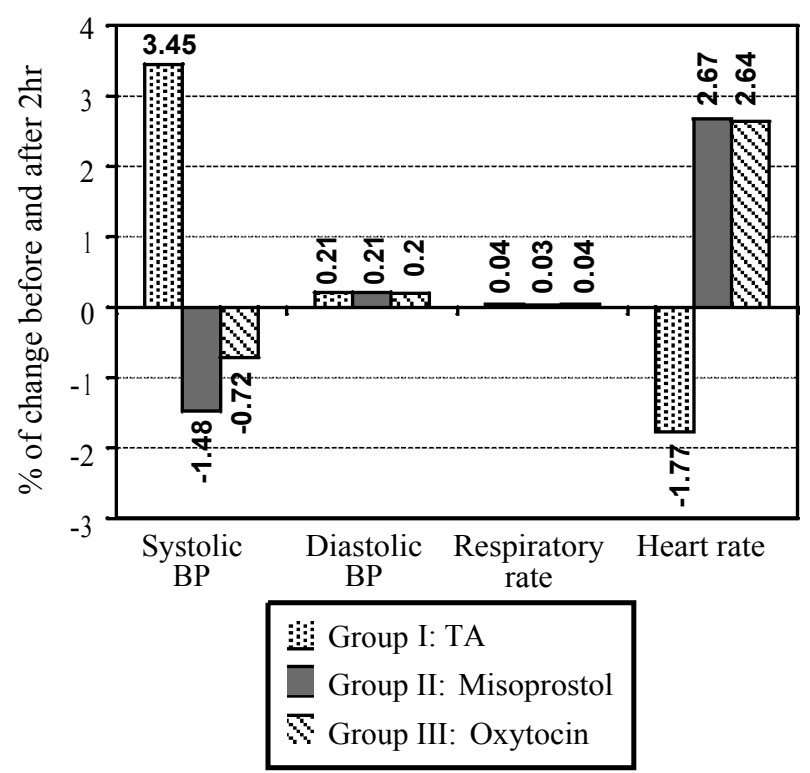

Fig. (9): Bar chart between groups according to $\%$ of change before and after $2 \mathrm{hrs}$.
Table (8): Comparison between groups according to laboratory data before delivery.

\begin{tabular}{|c|c|c|c|c|c|}
\hline $\begin{array}{l}\text { Laboratory data } \\
\text { before delivery }\end{array}$ & $\begin{array}{l}\text { Group I: } \\
\text { TA } \\
(\mathrm{N}=50)\end{array}$ & $\begin{array}{c}\text { Group II: } \\
\text { Misoprostol } \\
(\mathrm{N}=50)\end{array}$ & $\begin{array}{c}\text { Group III: } \\
\text { Oxytocin } \\
(\mathrm{N}=50)\end{array}$ & $\mathrm{F}$ & $\begin{array}{c}p- \\
\text { value }\end{array}$ \\
\hline $\mathrm{Hb} \%$ & $\begin{array}{l}11.40 \pm \\
3.19\end{array}$ & $\begin{array}{l}11.02 \pm \\
3.09\end{array}$ & $\begin{array}{l}10.80 \pm \\
3.02\end{array}$ & 0.674 & 0.471 \\
\hline HCT & $\begin{array}{l}34.00 \pm \\
9.52\end{array}$ & $\begin{array}{l}36.00 \pm \\
10.08\end{array}$ & $\begin{array}{l}35.00 \pm \\
9.80\end{array}$ & 0.551 & 0.690 \\
\hline INR & $\begin{array}{l}1.05 \pm \\
0.29\end{array}$ & $\begin{array}{l}1.10 \pm \\
0.31\end{array}$ & $\begin{array}{l}1.02 \pm \\
0.29\end{array}$ & 0.455 & 0.610 \\
\hline Prothrombin time & $\begin{array}{l}14.00 \pm \\
3.92\end{array}$ & $\begin{array}{l}15.00 \pm \\
4.20\end{array}$ & $\begin{array}{l}13.00 \pm \\
3.64\end{array}$ & 0.741 & 0.462 \\
\hline SGOT & $\begin{array}{l}20.00 \pm \\
5.60\end{array}$ & $\begin{array}{l}22.00 \pm \\
6.16\end{array}$ & $\begin{array}{l}19.00 \pm \\
5.32\end{array}$ & 0.606 & 0.669 \\
\hline SGPT & $\begin{array}{l}30.00 \pm \\
8.40\end{array}$ & $\begin{array}{l}31.00 \pm \\
8.68\end{array}$ & $\begin{array}{l}30.00 \pm \\
8.40\end{array}$ & 0.500 & 0.592 \\
\hline Billirubin & $\begin{array}{l}0.80 \pm \\
0.22\end{array}$ & $\begin{array}{l}0.82 \pm \\
0.23\end{array}$ & $\begin{array}{l}0.78 \pm \\
0.22\end{array}$ & 0.815 & 0.448 \\
\hline Urea & $\begin{array}{l}25.00 \pm \\
7.00\end{array}$ & $\begin{array}{l}25.50 \pm \\
7.14\end{array}$ & $\begin{array}{l}24.25 \pm \\
6.79\end{array}$ & 0.666 & 0.649 \\
\hline Creatinine & $\begin{array}{l}0.90 \pm \\
0.25\end{array}$ & $\begin{array}{l}0.92 \pm \\
0.26\end{array}$ & $\begin{array}{l}0.87 \pm \\
0.24\end{array}$ & 0.550 & 0.574 \\
\hline
\end{tabular}

F: ANOVA test. $\quad p$-value $>0.05$ NS

Table (8) shows no statistically significant difference between groups according to laboratory data before delivery.

Table (9): Comparison between groups according to laboratory data (on 2 nd days).

\begin{tabular}{|c|c|c|c|c|c|}
\hline $\begin{array}{l}\text { Laboratory data } \\
\text { (On 2nd days) }\end{array}$ & $\begin{array}{c}\text { Group I: } \\
\text { TA } \\
(\mathrm{N}=50)\end{array}$ & $\begin{array}{l}\text { Group II: } \\
\text { Misoprostol } \\
(\mathrm{N}=50)\end{array}$ & $\begin{array}{c}\text { Group III: } \\
\text { Oxytocin } \\
(\mathrm{N}=50)\end{array}$ & $\mathrm{F}$ & $\begin{array}{c}p- \\
\text { value }\end{array}$ \\
\hline $\mathrm{Hb} \%$ & $\begin{array}{l}10.94 \pm \\
3.06\end{array}$ & $\begin{array}{l}10.58 \pm \\
2.96\end{array}$ & $\begin{array}{l}10.48 \pm \\
2.93\end{array}$ & 0.761 & 0.533 \\
\hline $\mathrm{HCT}$ & $\begin{array}{l}32.64 \pm \\
9.14\end{array}$ & $\begin{array}{l}34.56 \pm \\
9.68\end{array}$ & $\begin{array}{l}33.95 \pm \\
9.51\end{array}$ & 0.622 & 0.780 \\
\hline Total blood loss & $\begin{array}{l}539.45 \pm \\
22.38\end{array}$ & $\begin{array}{l}517.40 \pm \\
21.61\end{array}$ & $\begin{array}{l}548.90 \pm \\
22.71\end{array}$ & 0.324 & 0.295 \\
\hline INR & $\begin{array}{l}1.09 \pm \\
0.31\end{array}$ & $\begin{array}{l}1.14 \pm \\
0.32\end{array}$ & $\begin{array}{l}1.06 \pm \\
0.30\end{array}$ & 0.514 & 0.689 \\
\hline Prothrombin time & $\begin{array}{l}13.44 \pm \\
3.76\end{array}$ & $\begin{array}{l}14.40 \pm \\
4.03\end{array}$ & $\begin{array}{l}12.61 \pm \\
3.53\end{array}$ & 0.837 & 0.522 \\
\hline SGOT & $\begin{array}{l}19.20 \pm \\
5.38\end{array}$ & $\begin{array}{l}21.12 \pm \\
5.91\end{array}$ & $\begin{array}{l}18.43 \pm \\
5.16\end{array}$ & 0.684 & 0.756 \\
\hline SGPT & $\begin{array}{l}28.80 \pm \\
8.06\end{array}$ & $\begin{array}{l}29.76 \pm \\
8.33\end{array}$ & $\begin{array}{l}29.10 \pm \\
8.15\end{array}$ & 0.565 & 0.669 \\
\hline Billirubin & $\begin{array}{l}0.77 \pm \\
0.22\end{array}$ & $\begin{array}{l}0.78 \pm \\
0.22\end{array}$ & $\begin{array}{l}0.75 \pm \\
0.21\end{array}$ & 0.921 & 0.507 \\
\hline Urea & $\begin{array}{l}24.00 \pm \\
6.72\end{array}$ & $\begin{array}{l}24.48 \pm \\
6.85\end{array}$ & $\begin{array}{l}23.52 \pm \\
6.59\end{array}$ & 0.753 & 0.733 \\
\hline Creatinine & $\begin{array}{l}0.86 \pm \\
0.24\end{array}$ & $\begin{array}{l}0.88 \pm \\
0.25\end{array}$ & $\begin{array}{l}0.85 \pm \\
0.24\end{array}$ & 0.622 & 0.649 \\
\hline
\end{tabular}

F: ANOVA test. $p$-value $>0.05 \mathrm{NS}$

Estimated blood loss = EBV x (Preop heamtorcit - Postop hematocrit) I Preop hematocrit

Estimated blood volume in $\mathrm{mL}=$ Women's weight in $\mathrm{kg} \mathrm{x} 85$

Table (9) shows no statistically significant difference between groups according to laboratory data on ( 2 nd day). 
Table (10): Comparison between groups according to $\%$ of change in laboratory data.

\begin{tabular}{lllllll}
\hline \% of change in & $\begin{array}{c}\text { Group I: } \\
\text { TA } \\
\text { laboratory data } \\
(\mathrm{N}=50)\end{array}$ & $\begin{array}{c}\text { Group II: } \\
\text { Misoprostol } \\
(\mathrm{N}=50)\end{array}$ & $\begin{array}{c}\text { Group III: } \\
\text { Oxytocin } \\
(\mathrm{N}=50)\end{array}$ & $\mathrm{F}$ & $\begin{array}{c}p \text { - } \\
\text { value }\end{array}$ \\
\hline $\mathrm{Hb} \%$ & $0.46 \pm$ & $0.44 \pm$ & $0.32 \pm$ & 0.860 & 0.602 \\
& 0.13 & 0.12 & 0.09 & & \\
$\mathrm{HCT}$ & $1.36 \pm$ & $1.44 \pm$ & $1.05 \pm$ & 0.602 & 0.421 \\
& 0.38 & 0.40 & 0.29 & & \\
INR & $-0.04 \pm$ & $-0.04 \pm$ & $-0.04 \pm$ & 0.703 & 0.881 \\
& 0.01 & 0.01 & 0.01 & & \\
Prothrombin time & $0.56 \pm$ & $0.60 \pm$ & $0.39 \pm$ & 0.581 & 0.779 \\
& 0.16 & 0.17 & 0.11 & & \\
SGOT & $0.80 \pm$ & $0.88 \pm$ & $0.57 \pm$ & 0.946 & 0.590 \\
& 0.22 & 0.25 & 0.16 & & \\
SGPT & $1.20 \pm$ & $1.24 \pm$ & $0.90 \pm$ & 0.773 & 0.854 \\
& 0.34 & 0.35 & 0.25 & & \\
Billirubin & $0.03 \pm$ & $0.03 \pm$ & $0.02 \pm$ & 0.639 & 0.756 \\
& 0.01 & 0.01 & 0.01 & & \\
Urea & $1.00 \pm$ & $1.02 \pm$ & $0.73 \pm$ & 1.041 & 0.572 \\
& 0.28 & 0.29 & 0.20 & & \\
Creatinine & $0.04 \pm$ & $0.04 \pm$ & $0.03 \pm$ & 0.851 & 0.829 \\
& 0.01 & 0.01 & 0.01 & & \\
\hline
\end{tabular}

F: ANOVA test. $\quad p$-value $>0.05$ NS.

Table (10) shows no statistically significant difference between groups according to $\%$ of change in laboratory data.

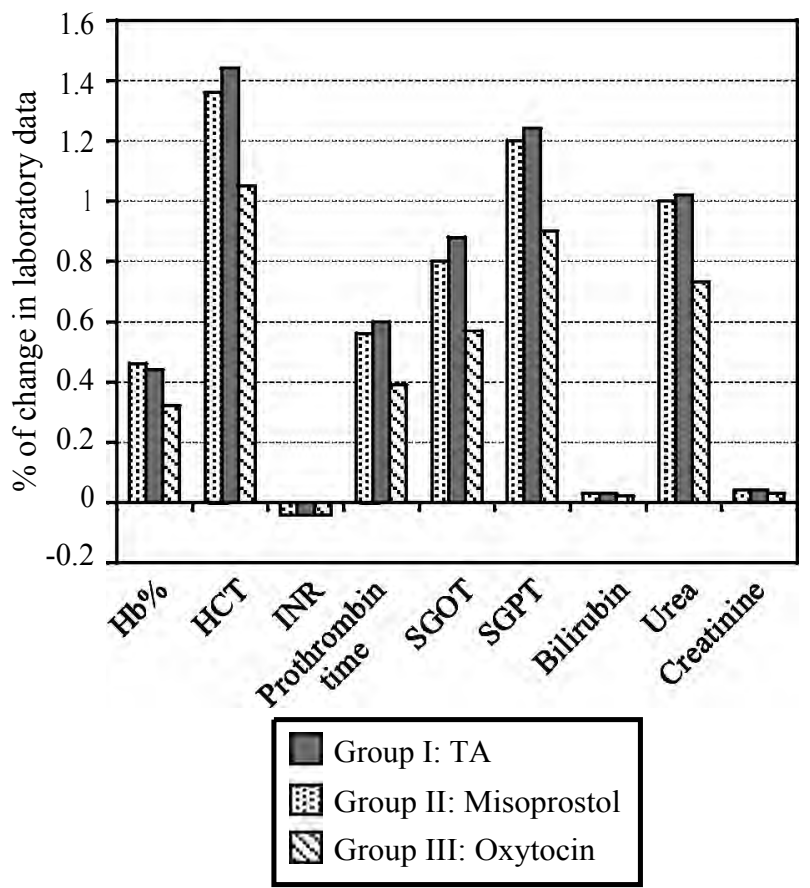

Fig. (10): Bar chart between groups according to \% of change in laboratory data.

Table (11): Comparison between groups according to neonatal manifestations.

\begin{tabular}{llllll}
\hline $\begin{array}{l}\text { Neonatal } \\
\text { manifestations }\end{array}$ & $\begin{array}{c}\text { Group I: } \\
\text { TA } \\
(\mathrm{N}=50)\end{array}$ & $\begin{array}{c}\text { Group II: } \\
\text { Misoprostol } \\
(\mathrm{N}=50)\end{array}$ & $\begin{array}{c}\text { Group III: } \\
\text { Oxytocin } \\
(\mathrm{N}=50)\end{array}$ & Test & $\begin{array}{c}p \text { - } \\
\text { value }\end{array}$ \\
\hline Apgar score 1min. & $8(2)$ & $8(1)$ & $8(2)$ & 0.271 & 0.582 \\
Apgar score 5min. & $8(2)$ & $9(1)$ & $9(1)$ & 0.293 & 0.682 \\
Weight (g) & $\begin{array}{l}3249.17 \pm \\
433.29\end{array}$ & $\begin{array}{l}3119.20 \pm \\
385.63\end{array}$ & $\begin{array}{l}3184.19 \pm \\
424.62\end{array}$ & 0.192 & 0.443 \\
& 4 & & & \\
\hline
\end{tabular}

$\mathrm{x}^{2}$ : Chi-square test. F: ANOVA test. $p$-value $>0.05$ NS.
Table (11) shows no statistically significant difference between groups according to neonatal manifestations.

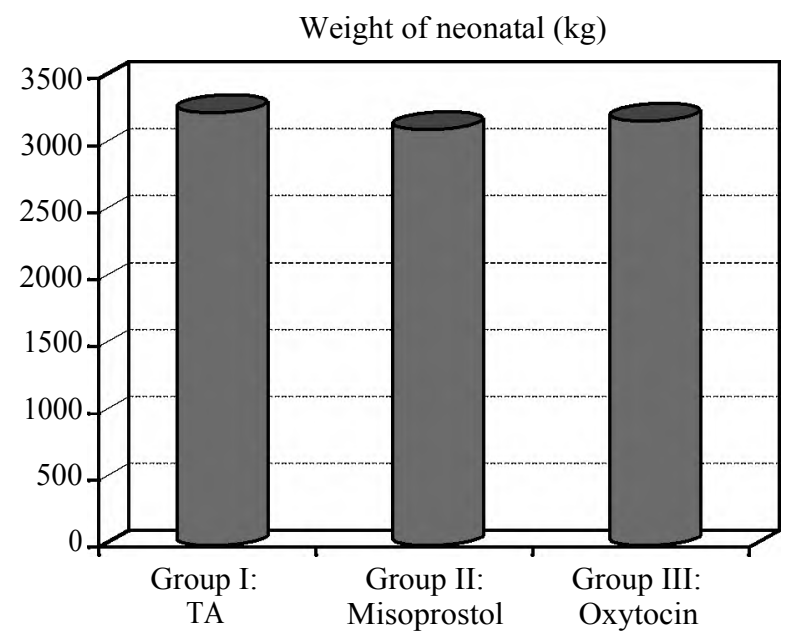

Fig. (11): Bar chart between groups according to weight of neonatal $(\mathrm{kg})$.

\section{Discussion}

Obstetric hemorrhage remains one of the major causes of maternal death in both developed and developing countries. Because of its importance as a leading cause of maternal mortality and morbidity, and because of evidence of substandard care in the majority of fatal cases, obstetric hemorrhage must be considered as a priority topic for national research development.

The increased frequency of PPH in the developing world is mainly due to expectant management because of lack of availability of medications used in the active management of the third stage [14].

Tranexamic acid competitively inhibits activation of plasminogen, thereby reducing conversion of plasminogen to plasmin (fibrinolysin), an enzyme that degrades fibrin clots, fibrinogen, and other plasma proteins, including the procoagulant factors V and VIII. Tranexamic acid also directly inhibits plasmin activity, but higher doses are required than are needed to reduce plasmin formation. In vitro, the antifibrinolytic potency of tranexamic acid is approximately 5 to 10 times that of aminocaproic acid [15]. It was used in gynecological bleeding and major trauma.

This study was held to assess the effectiveness of tranexamic acid in reducing blood loss in patients undergoing cesarean sections.

Our results showed that tranexamic acid significantly reduces bleeding during and after cesarean 
section. These results agreed with the results of the three mentioned studies. There was significant statistical difference $(p$-value $<0.05)$ in the vital data immediately after placental delivery between 3 groups upper hand for oxytocin and no significant difference in the vital data 2 hours postoperative between the 3 groups. Postoperative Hemoglobin was no significant difference in 3 groups. These results support other studies.

In this study total blood loss from placental delivery until end of cesarean section was significantly lower in 3 groups. Blood loss from placental delivery till 2 hours post-operative was reported whatever it is. There was no significant difference in vital data between the 3 groups 2 hours postoperative.

In this study the included patients were those who are term, going for elective cesarean section, and excluded Major maternal medical problem, Patients with bleeding tendency, Patient with high risk of thromboembolism, Ante-partum hemorrhage, Abnormal site of the placenta. We included all parities not only the primipara.

Possible bias in our study might result from exclusion of the cases with higher risks for $\mathrm{PPH}$, but they were also excluded from the other studies.

In this study there is no statistical difference between groups as regard intraoperative events as accessory haemostatic sutures in the uterine incision, uterine artery injuries or broad ligament hematoma.

No cases needed blood transfusion in the study group, while only 1 case in the control group required blood transfusion, and that difference was not of statistical significance.

No cases needed hysterectomy in neither the study group nor the control group.

Only few cases were reported to show minor side effects as nausea, vomiting and headache in the study group.

No cases were reported to show thrombotic events within one week postoperative.

Neonatal outcomes were not affected in both groups.

\section{Conclusion:}

The use of tranexamic acid prior to cesarean section seems to be effective (in addition to its low cost) in reduction of blood loss during and after cesarean section.
All data demonstrated that tranexamic acid can be used safely to reduce bleeding during and after CS and its use was not associated with any maternal and neonatal side effects by mentioned dose.

\section{References}

1- LU M.C., FRIDMAN M., KORST L.M., GREGORY K.D., REYES C., HOBEL C.J., et al.: Variations in the incidence of postpartum haemorrhageacross hospitals in California. Matern Child Health J., 9: 297-306, 2005.

2- MAGANN E.F., EVANS S., HUTCHINSON M., COLLINS R., LANNEAU G. and MORRISON J.C.: Postpartum hemorrhage after cesareandelivery: An analysis of risk factors. South Med. J., 98: 681-685, 2005.

3- GÜNGÖRDÜK K., ASICIOGLU O., CELIKKOL O., OLGAC Y. and ARK C.: Use of additional oxytocin to reduce blood loss at electivecaesarean section: A randomised control trial. Aust. N. Z. JObstet. Gynaecol., 50: 36-39, 2010.

4- BOLTON T.J., RANDALL K. and YENTIS S.M.: Effect of the confidential enquiries into maternal deaths on the use of syntocinon at caesarean section in the UK. Anaesthesia, 58: 277-279, 2003.

5- GLEESON N.C., BUGGY F., SHEPPARD B.L. and BONNAR J.: The effect of tranexamic acid on measured menstrual loss and endometrial fibrinolytic enzymes in dysfunctional uterine bleeding. Acta. Obstet. Gynecol. Scand, 73: 274-277, 1994.

6- DUNN C.J. and GOA K.L.: Tranexamic acid: A review of its use in surgery and other indications. Drugs, 57: 1005-1032, 1999.

7- NOVIKOVA N. and HOFMEYR G.J.: Tranexamic acid for preventing postpartum haemorrhage. Cochrane Database Syst. Rev., 7: CD007872, 2010.

8- PRENTICE C.R.: Basis of antifibrinolytic therapy. J. Clin. Pathol. Suppl. (R. Coll. Pathol.), 14: 35-40, 1980.

9- HELLGREN M.: Hemostasis during normal pregnancy and puerperium. Semin Thromb Hemost, 29: 125-30, 2003.

10- CORTET M., DENEUX-THARAUX C., DUPONT C., et al.: Association between fibrinogen level and severity of postpartum haemorrhage: Secondary analysis of a prospective trial. Br. J. Anaesth., 108: 984-9, 2012.

11- DUCLOY-BOUTHORS A-S., JUDE B., DUHAMEL A., et al.: High-dose tranexamicacid reduces blood loss in postpartum haemorrhage. Crit. Care, 15: R117, 2011.

12- ABOUZAHR C.: Antepartum and postpartum haemorrhage. In: Murray C.J., Lopez A.D., eds. Health Dimensions of Sex and Reproduction. Boston, Mass: Harvard University Press, 172-4, 1998.

13- CYKLOKAPRON (Pharmacia): In: Krogh C.M.E., editor CPS Compendium of pharmaceuticals and specialties. 22nd ed. Ottawa: Canadian Pharmaceutical Association, p. $211,1987$. 


\section{دورحمض الترانيكساميك فى ثقص فقدان الدم أثناء وبعد الولادة القيصرية بوائيط بواسطة}

معدل الولادات القيصرية فى تزايد ومتوبط الدم المفقود مع كل قيصرية ( . . . مل) يمثل ضعف متوسط فقدان الدم مع الولادة الطبيعية (0..)

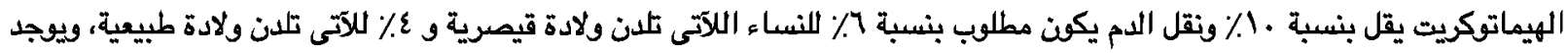

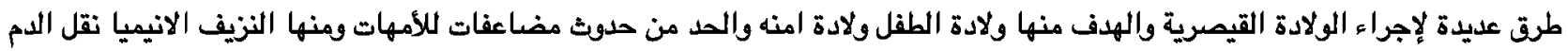

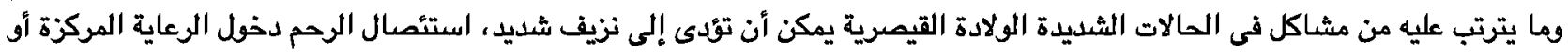
إلى وفاة الأم. بعض الأدوية مثل الاوكسيتوسين، الميثرجين، المينوبروستول. بروستاجلاندين قد استخدمت لعلاج هذا النزيف.

حامض الترانيكساميك هو عامل قوى يعمل كمضاد حل الفييربن عن طريق حجب مواقع الارتباط بالليسين على جزيئات البلازميتوجين حامض الترانيكساميك له القدرة على تحسين الية تخثر الدم داخل الجسم ومن ثم فهو يمنع تفتيت الجلطة بالتالى يقل كمية الدم المفقود .

يستخدم حامض ترانيكساميك للحد من فقدان الدم والحاجة لنقل الدم فى مجال جراحة القلب ونداعة الكبد والعمليات الجراحية العظمية

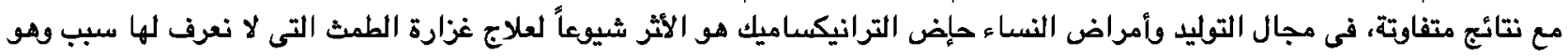

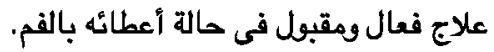

الدم المصاحب للحمل كحالات انفصال المشيمة والمشيمة المزاحة أيضاً يستخدم حإض الترانيكساميك كعلاج. هذه الدراسة هى دراسة عملية وبسوف تؤدى على • ما سيدة من قسم النساء والولادة مستشفى السيد جلال الجامعى ومستشفى الساحل التعليمى.

الاستتاجات: أن إعطاء حمض الترانيكساميك يقلل بلرجة مؤثرة الفاقد من الدم أثناء أو بعد العملية القيصرية وبالتالى ينصسح بإستخدمه بصورة دورية قبل العملية القيصرية. 